\title{
DENSITY THEOREMS FOR SAMPLING AND INTERPOLATION IN THE BARGMANN-FOCK SPACE III
}

\author{
SVEIN BREKKE AND KRISTIAN SEIP
}

\section{Introduction.}

This series of papers is devoted to what we call sets of sampling and interpolation for the Bargmann-Fock space of entire functions. Parts I and II [13, 14] contained a complete description of sets of this kind in terms of density properties of discrete sets in the complex plane. In this paper, Part III of the series, we show that these theorems allow natural extensions when we consider values (on a discrete set) of functions in the space as well as of their derivatives up to a certain order.

Similar problems have been studied by many authors. The idea of derivative sampling and interpolation is well-known from the theory of bandlimited functions (see [9, p. 1571]). Sampling of the type considered in this paper has been investigated by Unterberger, in a more general context but without sharp results (see [15, Theorem 8.1]). An interpolation problem for $H^{p}$ spaces involving derivatives was stated and solved by Øyma in [16].

We find derivative sampling and interpolation to be of particular interest in the Bargmann-Fock space, since there is a nice interpretation in the term of "phase space localization"; we shall comment further on this once we have stated our theorems.

Our proof techniques are basically the same as those in [14] (which means that Beurling's ideas from [3] play a central role). We shall therefore be relatively brief at many points and often refer to [14] and [3] for details.

\section{Results.}

For $\alpha>0$ let $d \mu_{\alpha}(\zeta)=\frac{\alpha}{\pi} e^{-\alpha|\zeta|^{2}} d \xi d \eta, \zeta=\xi+i \eta$, and define the Bargmann-Fock space $F_{\alpha}^{2}$ to be the collection of entire functions $f(\zeta)$ for which 


$$
\|f\|_{2}=\|f\|_{\alpha, 2}=\iint_{C}|f(\zeta)|^{2} d \mu_{\alpha}(\zeta)<\infty
$$

$F_{\alpha}^{2}$ is a Hilbert space with reproducing kernel $K(z, \zeta)=e^{\alpha z \zeta}$, i.e., for every $f \in F_{\alpha}^{2}$ we have

$$
f(z)=\langle f, K(z, \cdot)\rangle,
$$

the inner product being defined as

$$
\langle f, g\rangle=\langle f, g\rangle_{\alpha}=\iint_{c} f(\zeta) \overline{g(\zeta)} d \mu_{\alpha}(\zeta)
$$

The normalized reproducing kernels, $k_{z}(\zeta)=K(z, z)^{-\frac{1}{2}} K(z, \zeta)$ correspond, via the Bargmann transform (see below), to the canonical coherent states of quantum mechanics, and to Gabor wavelets in signal analysis.

Of basic importance is the fact that the translations

$$
\left(T_{z} f\right)(\zeta)=\left(T_{z}^{\alpha \zeta}\right)(\zeta)=e^{\alpha z \zeta-\frac{\alpha}{2}|z|^{2}} f(\zeta-z)
$$

act isometrically in $F_{\alpha}^{2}$. Note, e.g., that $k_{z}=T_{z} 1(1(\zeta) \equiv 1)$, and observe that (1) follows from an application of Cauchy's formula around 0 , the unitarity of the operator $T_{z}$, and the fact that $T_{z}^{-1}=T_{-z}$.

We recall from [11] that a discrete set $\Gamma$ of complex numbers in a set of sampling for $F_{\alpha}^{2}$ if there exist positive numbers $A$ and $B$ such that

$$
A\|f\|_{2}^{2} \leqq \sum_{z \in \Gamma} e^{-\alpha|z|^{2}}|f(z)|^{2}=\sum_{z \in \Gamma}\left|\left\langle f, T_{z} 1\right\rangle\right|^{2} \leqq B\|f\|_{2}^{2}
$$

for all $f \in F_{\alpha}^{2}$. If to every $l^{2}$-sequence $\left\{\alpha_{j}\right\}$ of complex numbers there exists an $f \in F_{\alpha}^{2}$ such that $e^{-\frac{\alpha}{x}\left|z_{j}\right| 2} f\left(z_{j}\right)=\left\langle f, T_{z_{j}} 1\right\rangle=a_{j}$ for all $j, \Gamma=\left\{z_{j}\right\}$ is said to be a set of interpolation for $F_{\alpha}^{2}$. A set of sampling corresponds, in the terminology of [5], to a frame of coherent states.

There is a natural extension of (1) to "translation invariant" differentiation, to be described next. It follows from Cauchy's formula that evaluation of the $k$ th derivative at 0 is a bounded linear functional given by the formula

$$
f^{(k)}(0)=\left(\alpha^{k} k !\right)^{\frac{1}{2}}\left\langle f, f_{k}\right\rangle,
$$

where $f_{k}$ is the unit vector

$$
f_{k}(z)=\left(\frac{\alpha^{k}}{k !}\right)^{\frac{1}{2}} z^{k}
$$

At an arbitrary point $\zeta$ we then associate the inner products $\left\langle f, T_{\zeta} f_{k}\right\rangle$ (instead of the derivatives themselves). From (3) we easily find that 


$$
\left\langle f, T_{z} f_{k}\right\rangle=\left(\alpha^{k} k !\right)^{-\frac{1}{2}} e^{-\frac{\alpha}{2}|z|^{2}} \sum_{j=0}^{k}\left(\begin{array}{l}
k \\
j
\end{array}\right)(-\alpha \bar{z})^{(k-j)} f^{(j)}(z),
$$

so there is a one-to-one correspondence between $f(z), f^{\prime}(z), \ldots, f^{(k)}(z)$ and the inner products $\left\langle f, T_{z} f_{0}\right\rangle,\left\langle f, T_{z} f_{1}\right\rangle, \ldots,\left\langle f, T_{z} f_{k}\right\rangle$.

Let now $\Gamma=\left\{z_{j}\right\}_{j=1}^{\infty}$ be a sequence of distinct points from $C$. To this set we associate a "multiplicity function", $v: \Gamma \rightarrow \mathrm{N}$, and we construct a sequence of points, $\Gamma_{v}$, consisting of the points from $\Gamma$ with each $z$ from $\Gamma$ appearing $v(z)$ times in the sequence. If $\Gamma^{\prime}$ is a subset of $\Gamma, \Gamma_{v}^{\prime}$ denotes the sequence where each point $z^{\prime}$ from $\Gamma^{\prime}$ is listed $v\left(z^{\prime}\right)$ times. We shall say that $\Gamma_{v}$ is a set of sampling for $F_{\alpha}^{2}$ if there exist positive numbers $A$ and $B$ such that

$$
A\|f\|_{2}^{2} \leqq \sum_{j=1}^{\infty} \sum_{k=0}^{v\left(z_{j}\right)-1}\left|\left\langle f, T_{z_{j}} f_{k}\right\rangle\right|^{2} \leqq B\|f\|_{2}^{2}
$$

for all $f \in F_{\alpha}^{2}$. If to every sequence $\left\{\alpha_{j}^{(k)}\right\}$ for which

$$
\sum_{j=1}^{\infty} \sum_{k=0}^{v\left(z_{j}\right)-1}\left|\alpha_{j}^{(k)}\right|^{2}<\infty
$$

there exists an $f \in F_{\alpha}^{2}$ such that $\left\langle f, T_{z_{j}} f_{k}\right\rangle=\alpha_{j}^{(k)}$ for all $j$ and $0 \leqq k \leqq v(j)-1, \Gamma_{v}$ is said to be a set of interpolation for $F_{\alpha}^{2}$.

As in Parts I and II, we use Landau's generalization of Beurling's notion of uniform densities [10]. We consider then uniformly discrete sets, i.e., discrete sets $\Gamma=\left\{z_{j}\right\}$ for which $q=q(\Gamma)=\inf _{j-k}\left|z_{j}-z_{k}\right|>0 ; q$ will be referred to as the separating distance of $\Gamma$. We assume also that $\sup _{z \in \Gamma} v(z)<\infty$. Fix a compact set $I$ of measure 1 in the complex plane, whose boundary has measure 0 . Let $n^{-}(r)$ and $n^{+}(r)$ denote respectively the smallest and largest number of points from $\Gamma_{v}$ to be found in a translate of $r I$. We define the lower and upper uniform densities of $\Gamma_{v}$ to be

$$
D^{-}\left(\Gamma_{v}\right)=\liminf _{r \rightarrow \infty} \frac{n^{-}(r)}{r^{2}} \text { and } D^{+}\left(\Gamma_{v}\right)=\limsup _{r \rightarrow \infty} \frac{n^{+}(r)}{r^{2}},
$$

respectively. It was proved by Landau that these limits are independent of $I$ (Landau's arguments apply since $\sup ^{\nu}(z)<\infty$ ).

Our main theorems are the following.

THEOREM 2.1. Let $\sup _{z \in \Gamma} v(z)<\infty$. Then $\Gamma_{v}$ is a set of sampling for $F_{\alpha}^{2}$ if and only if $\Gamma$ can be expressed as a finite union of uniformly discrete sets and contains a uniformly discrete subset $\Gamma^{\prime}$ for which $D^{-}\left(\Gamma_{v}^{\prime}\right)>\frac{\alpha}{\pi}$. 
THEOREM 2.2. Let $\sup _{z \in \Gamma} v(z)<\infty$. Then $\Gamma_{v}$ is a set of interpolation for $F_{\alpha}^{2}$ if and only if $\Gamma$ is uniformly discrete and $D^{+}\left(\Gamma_{v}\right)<\frac{\alpha}{\pi}$.

We will need to consider the corresponding $L^{\infty}$ problem. Let then $F_{\alpha}^{\infty}$ denote the Banach space consisting of those entire functions $f(z)$ for which

$$
\|f\|_{\infty}=\|f\|_{\alpha, \infty}=\sup _{z} e^{-\frac{\alpha}{2}|z|^{2}}|f(z)|<\infty .
$$

We denote that the translations $T_{z}$ act isometrically in $F_{\alpha}^{\infty}$ as wel, and observe that the integrals $\left\langle f, T_{z} f_{k}\right\rangle$ are still meaningful, We say that $\Gamma_{v}$ is a set of sampling for $F_{\alpha}^{\infty}$ if there exists a positive number $K$ such that

$$
\|f\|_{\infty} \leqq K \sup _{z \in \Gamma, 0 \leqq k \leqq v(z)-1}\left|\left\langle f, T_{z} f_{k}\right\rangle\right|
$$

for all $f \in F_{\alpha}^{\infty}$. If to every bounded sequence $\left\{a_{j}^{(k)}\right\}$ there exists an $f \in F_{\alpha}^{\infty}$ such that $\left\langle f, T_{z_{j}} f_{k}\right\rangle=a_{j}^{(k)}$ for all $j$ and $0 \leqq k \leqq v\left(z_{j}\right)-1$, we say that $\Gamma_{v}$ is a set of interpolation for $F_{\alpha}^{\infty}$.

We have then the following theorems.

THEOREM 2.3. Let $\sup _{z \in \Gamma} v(z)<\infty$. Then $\Gamma_{v}$ is a set of sampling for $F_{\alpha}^{\infty}$ if and only if $\Gamma$ contains a uniformly discrete subset $\Gamma^{\prime}$ for which $D^{-}\left(\Gamma_{v}^{\prime}\right)>\frac{\alpha}{\pi}$.

THEOREM 2.4. Let $\sup _{z \in \Gamma} v(z)<\infty$. Then $\Gamma_{v}$ is a set of interpolation for $F_{\alpha}^{\infty}$ if and only if $\Gamma$ is uniformly discrete and $D^{+}\left(\Gamma_{v}\right)<\frac{\alpha}{\pi}$.

REMARK 1. $f(x+i y), f \in F_{1 / 2}^{2}$, can be thought of as representing a signal $s$ at time $x$ and frequency $y$ : The transformation $s \mapsto f$ given by

$$
f(z)=e^{\frac{1}{2}|z|^{2}} e^{i \frac{x y}{2}} \int_{-\infty}^{\infty} s(t) g(x-t) e^{i y t} d t,
$$

with $g(t)=\exp \left(-\frac{\mathrm{t}^{2}}{2}\right)(c$ an appropriate constant), is a unitary map (the Bargmann transform) from $L^{2}(\mathrm{R})$ into $F_{1 / 2}^{2}$. It is well-known and easy to check that the functions $f_{k}(z)$ are the images of the Hermite functions $H_{k}(t)$ under the Bargmann transform [6, p. 39]. $e^{-i x t} H_{k}(t-y), k \leqq n-1$, are the $n$ functions best localized around time $x$ and frequency $y$ in the following sense: $T_{z} f_{k}$ is the function in $\operatorname{span}\left\{T_{z} f_{m}\right\}_{m<k}^{\perp}$ that maximizes the concentration

$$
\lambda(f)=\frac{\iint_{|z-\zeta|<R}|f(\zeta)|^{2} d \mu_{\alpha}(\zeta)}{\iint_{R}|f(\zeta)|^{2} d \mu_{\alpha}(\zeta)}
$$


for any $R>0$. Thus, to each point $z$ of our discrete set $\Gamma$, we associate the $v(z)$ functions that are optimally concentrated around that point.

For more on this aspect of time-frequency (or phase space) localization, see [3, pp. 14-18], $[4,11]$.

REMARK 2. It follows from Theorems 2.1 and 2.2 above that for no sequences $\left\{t_{j}\right\},\left\{w_{j}\right\}$ of real numbers will the functions $e^{-i w_{j}} H_{k}\left(t-t_{j}\right), 0 \leqq k \leqq n-1$ for some $n \geqq 0$, constitute a Riesz basis for $L^{2}(R)$. This we pointed out in [11] for $n=1$, and we observe now that it did not help us to bring in the first few Hermite functions. This aspect of our theorems seem to reflect in yet another way the "Balian-Low phenomenon" (see [1]). Note, however, that Gröchenig and Walnut have found an interesting Riesz basis related to sampling and interpolation [7]; this is one of many recent papers on "how to beat" the Balian-Low theorem.

REMARK 3. In the context of bandlimited functions we may also give a description in terms of densities in much the same way as above with, however, two notable differences: Firstly, we will not have the nice time-frequency interpretation, and secondly, the description will not be complete since there we may find sets of both sampling and interpolation.

REMARK 4. The density theorems for Bergman spaces proved in [12] can be extended to theorems similar to those stated above (this we leave to the interested reader to verify).

Remark 5. We may ask what happens if $v\left(z_{j}\right) \rightarrow \infty$ as $\left|z_{j}\right| \rightarrow \infty$. Can we construct Riesz bases if $v\left(z_{j}\right)$ grows sufficiently fast?

\section{Preliminaries.}

Let $\Gamma=\left\{z_{j}\right\}$ and an associated "multiplicity function" $v$ be given. We define $\max (v)=\sup _{z \in \Gamma} v(z)$, which for the rest of the paper is assumed to be finite. We put

$$
S\left(\Gamma_{v}\right) f=S_{\alpha}\left(\Gamma_{v}\right) f=\left\{\left\langle f, T_{z_{j}} f_{k}\right\rangle_{\alpha}: z_{j} \in \Gamma, 0 \leqq k \leqq v\left(z_{j}\right)-1\right\}
$$

Then

$$
\left\|S\left(\Gamma_{\phi}\right) f\right\|_{\infty}=\sup _{z \in \Gamma, 1 \leqq k \leqq v(z)-1}\left|\left\langle f, T_{z} f_{k}\right\rangle\right|
$$

and similarly,

$$
\left\|S\left(\Gamma_{v}\right) f\right\|_{2}=\left(\sum_{z \in \Gamma} \sum_{k=0}^{v(z)-1}\left|\left\langle f, T_{z} f_{k}\right\rangle\right|^{2}\right)^{\frac{1}{2}}
$$


$M_{p}\left(\Gamma_{v}\right)=M_{p}\left(\Gamma_{v}, \alpha\right)(p=2$ or $p=\infty)$ will denote the smallest number $M_{p}$ such that

$$
\|f\|_{p} \leqq M_{p}\left\|S\left(\Gamma_{v}\right) f\right\|_{p}
$$

for all $f \in F_{\alpha}^{p}$. Hence $\Gamma_{v}$ is a set of sampling for $F_{\alpha}^{\infty}$ if and only if $M_{\infty}\left(\Gamma_{v}\right)<\infty$, and it is a set of sampling for $F_{\alpha}^{2}$ if and only if $M_{2}\left(\Gamma_{v}\right)<\infty$ and $\left\|S\left(\Gamma_{v}\right) f\right\|_{2}<\infty$ for all $f \in F_{\alpha}^{2}$.

If $\Gamma_{v}$ is a set of interpolation for $F_{\alpha}^{p}$, a standard argument based on the closed graph theorem [8, p. 196] shows that the interpolation can be performed in a stable way. This means that there exists a positive number $N_{p}$ such that for every $l^{p}$-sequence $\left\{a_{j}^{(k)}\right\}$ we can find an $f \in F_{\alpha}^{p}$ with $\left\langle f, T_{z_{j}} f_{k}\right\rangle=a_{j}^{(k)}$ for all $j$ and $0 \leqq k \leqq v\left(z_{j}\right)-1$, and

$$
\|f\|_{p} \leqq N_{p}\left\|S\left(\Gamma_{v}\right) f\right\|_{p} .
$$

The smallest such $N_{p}$ is denoted $N_{p}\left(\Gamma_{v}\right)=N_{p}\left(\Gamma_{v}, \alpha\right)$, and we put $N_{p}\left(\Gamma_{v}\right)=\infty$ if $\Gamma$ is not a set of interpolation for $F_{\alpha}^{p}$.

We recall the compactness property of $F_{\alpha}^{p}$ : If $\left\{f_{n}\right\}$ is a sequence in the ball

$$
\left\{f \in F_{\alpha}^{p}:\|f\|_{p} \leqq R\right\},
$$

then there is a subsequence $\left\{f_{n_{k}}\right\}$ converging pointwise and uniformly on compact sets to some function in the ball. This is immediate from the definition of $F_{\alpha}^{p}$ and a normal family argument.

A sequence $Q_{j}$ of closed sets converges strongly to $Q$, denoted $Q_{j} \rightarrow Q$, if $\left[Q, Q_{j}\right] \rightarrow 0$; here $[\cdot, \cdot]$ denotes the Fréchet distance between two closed sets. $Q_{j}$ converges weakly to $Q$, denoted $Q_{j} \rightarrow Q$, if for every compact set $D\left(Q_{j} \cap D\right) \cup \partial D \rightarrow(Q \cap D) \cup \partial D$. (Note that in our definition of weak limits we have eliminated an obvious error in Beurling's notes. Unfortunately, this error appears in [11] as well. The definition of weak limits in [11] should therefore be replaced by the present one.)

We now extend this notion to sequences $\Gamma_{v_{j}}^{(j)}$. We assume then that the sets $\Gamma^{(j)}$ are all uniformly discrete with a lower positive bound on their separating distances. Then $\Gamma^{(j)} \rightarrow \Gamma$ is equivalent to $z_{k}^{(j)} \rightarrow z_{k}$ for all $k$, provided the sets are appropriately indexed, $\Gamma^{(j)}=\left\{z_{k}^{(j)}\right\}, \Gamma=\left\{z_{k}\right\}$. We assume such an indexation and say that $\Gamma_{v_{j}}^{(j)} \rightarrow \Gamma_{v}$ if $\Gamma^{(j)} \rightarrow \Gamma$ and also $v_{j}\left(z_{k}^{(j)}\right) \rightarrow v\left(z_{k}\right)$ for all $k$. For a uniformly discrete set $\Gamma$ and its associated function $v$ we let $W\left(\Gamma_{v}\right)$ denote the collection of weak limits of the translated sequences $\Gamma_{v}+z$ (defined as $\Gamma_{v}+z=$ $\left\{\gamma_{1}+z, \gamma_{2}+z, \ldots\right\}$ for $\left.\Gamma_{v}=\left\{\gamma_{1}, \gamma_{2}, \ldots\right\}\right)$.

We shall need the following a generalization of Lemma 3.1 in [11]. 
LEMMA 3.1. For every nonnegative integer $k$ there is a constant $C=C(\alpha, k)$ such that

$$
\left\|\langle f , T _ { z } f _ { k } \rangle \left|-\left\langle f, T_{\zeta} f_{k}\right\rangle\|\leqq C|z-\zeta|\| f \|_{\infty}\right.\right.
$$

whenever $f \in F_{\alpha}^{\infty}$ and $|z-\zeta| \leqq 1$.

PROoF. This follows from Cauchy's formula and the translation invariance (see the proof of Lemma 3.1 in [11]).

The following local estimates are deduced in a similar fashion; here and in the sequel $D(z, r)$ denotes the disk of radius $r$ centered at $z$.

LEMMA 3.2. For every $f \in F_{\alpha}^{\infty}$ and $z \in \mathrm{C}$ we have

and

$$
\left|\left\langle f, T_{z} f_{k}\right\rangle\right| \leqq C_{1}(\alpha, r, k) \sup _{\zeta \in D(z, r)} e^{-\frac{\alpha}{2}|\zeta|^{2}}|f(\zeta)|
$$

$\quad\left|\left\langle f, T_{z} f_{k}\right\rangle\right|^{2} \leqq C_{2}(\alpha, r, k) \iint_{D(z, r)}|f(\zeta)|^{2} d \mu_{\alpha}(\zeta)$.

We finally recall a type of estimate from [14]. Let $\Lambda=\left\{\lambda_{m n}\right\}$ denote a square lattice, that is

$$
\lambda_{m n}=\sqrt{\pi / \alpha}(m+i n)
$$

for all integers $m, n$ and some positive number $\alpha, \alpha / \pi$ is referred to as the density of $\Lambda$. $Z=\left\{z_{m n}\right\}$ is uniformly close to $\Lambda$ if there exists a positive number $Q$ such that

$$
\left|z_{m n}-\lambda_{m n}\right| \leqq Q
$$

for all $m$ and $n$. If $\Gamma_{v}$ can be indexed so that it is uniformly close to a square lattice, say $\Gamma_{v}=\left\{\gamma_{m n}\right\}$, we associate a function $g(z)$ defined by

$$
g(z)=\left(z-\gamma_{00}\right)^{v\left(\gamma_{00}\right)} \prod_{\gamma_{m n} \neq \gamma_{00}}\left(1-\frac{z}{\gamma_{m n}}\right) \exp \left(\frac{\mathrm{z}}{\gamma_{m n}}+\frac{1}{2} \frac{\mathrm{z}^{2}}{\lambda_{m n}^{2}}\right)
$$

where $\gamma_{00}$ is the point of $\Gamma$ closest to 0 . The technique of estimation leading to Lemma 2.2 in [14] yields the following lemma.

LEMMA 3.3. Suppose $\Gamma_{v}$ can be indexed so that it is uniformly close to the square lattice $\Lambda$ of density $\alpha / \pi$, and let $g(z)$ be its associated function given by (9). Then there exist constants $C$ and $c$, depending on $\alpha, Q, q(\Gamma)$, and $\max (v)$, such that for every $z$ we have

$$
\left|e^{-\frac{\alpha}{2}|z|^{2}} g(z)\right| \leqq C e^{c|z| \log |z|}
$$




\section{Proof of Theorem 2.3.}

We check that some auxiliary results in Beurling's notes (also used in [11]) can be carried over to the present context.

LEMMA 4.1. If $\Gamma_{v}$ is a set of sampling for $F_{\alpha}^{\infty}$, then $\Gamma$ contains a uniformly discrete subset $\Gamma^{\prime}$ such that $\Gamma_{v}^{\prime}$ is also a set of sampling for $F_{\alpha}^{\infty}$.

Proof. As the proof of Theorem 2 in [3, p. 344], with Lemma 3.1 in place of Bernstein's theorem.

LEMMA 4.2. $\Gamma_{v_{j}}^{(j)} \rightarrow \Gamma_{v}$ and $\sup _{j} \max \left(v_{j}\right)<\infty$ imply $M_{\infty}\left(\Gamma_{v}\right) \leqq \liminf M_{\infty}\left(\Gamma_{v_{j}}^{(j)}\right)$.

Proof. (See the proof of Lemma 4.2 in [11].) For any $\varepsilon>0$ let $f \in F_{\alpha}^{\infty}$ be such that $\|f\|_{\infty}=1$ and $\left\|S\left(\Gamma_{v}\right) f\right\|_{\infty} \leqq M^{-1}+\varepsilon, M=M_{\infty}\left(\Gamma_{v}\right)$ (which may be infinite). We may assume that $|f(0)| \geqq 1-\varepsilon$. Now consider the function $f(a z), a<1$. We find that

$$
e^{-\frac{\alpha}{2}|z|^{2}}|f(a z)|=e^{-\frac{\alpha}{2}|a z|^{2}}|f(a z)| e^{-\frac{\alpha}{2}\left(1-a^{2}\right)|z|^{2}} .
$$

In view of Lemma 3.1, we have for $|z-a z|<C \varepsilon$ and $k \leqq \max (v)$ ( $C$ depending only on $\alpha$ and $\max (v))$,

$$
\left\|\left\langle f(a \cdot), T_{z} f_{k}\right\rangle|-|\left\langle f, T_{z} f_{k}\right\rangle\right\| \leqq \varepsilon .
$$

We choose $1-a=C \varepsilon^{3}$ so that for $|z| \geqq \varepsilon^{-2}-1$

$$
e^{-\frac{\alpha}{2}|z|^{2}}|f(a z)| \leqq e^{-\frac{C \alpha}{2 \varepsilon}\left(1-\varepsilon^{2}\right)^{2}}
$$

by (11). Thus for $|z| \geqq \varepsilon^{-2}$, or what is the same, $|z-a z| \geqq C \varepsilon$, we have

$$
\left|\left\langle f(a \cdot), T_{z} f_{k}\right\rangle\right| \leqq C(\alpha, k) e^{-\frac{C \alpha}{2 \varepsilon}\left(1-\varepsilon^{2}\right)}
$$

by (6). Then by the assumption on the sequence, we have for sufficiently small $\varepsilon$ and large $j,\left\|S\left(\Gamma_{v_{j}}^{(j)}\right) f(a \cdot)\right\|_{\infty} \leqq M^{-1}+3 \varepsilon$, or $M_{\infty}\left(\Gamma_{v_{j}}^{(j)}\right) \geqq(1-\varepsilon) /\left(M^{-1}+3 \varepsilon\right)$. Since $\varepsilon$ is arbitrary, the result follows.

There is an obvious generalization of the notion of sets of uniqueness. We shall say that $\Gamma_{v}$ is a set of uniqueness for $F_{\alpha}^{p}$ if $S\left(\Gamma_{v}\right) f=0$ implies that $f \equiv 0$. Note that (4) shows that this is equivalent to saying that if $f^{(k)}(z)=0$ for all $0 \leqq k \leqq v(z)-1, z \in \Gamma$, then $f \equiv 0$.

Lemma 4.3. Suppose $\Gamma$ is uniformly discrete. Then $M_{\infty}\left(\Gamma_{v}\right)<\infty$ if and only if every $\Gamma_{0} \in W\left(\Gamma_{v}\right)$ is a set of uniqueness for $F_{\alpha}^{\infty}$.

Proof. As the proof of Theorem 3 in [3, p. 345]. 
LEMMA 4.4. If $M_{\infty}\left(\Gamma_{v}, \alpha\right)<\infty$, then $M_{\infty}\left(\Gamma_{v}, \alpha+\varepsilon\right)<\infty$ for all sufficiently small $\varepsilon$.

Proof. As the proof of Theorem 4 in [3, p. 345].

PROOF OF THE NECESSITY PART OF TheOREM 2.3. We proceed as in the proof of the necessity part of Theorem 2.3 in [11]; the only difference is that the polynomial of Section 4 in [11] is replaced by a polynomial with zeros of order according to the value of the multiplicity function at each point in $\Gamma \cap D_{R}$. We will not repeat the details.

ProOf OF THE SUfFiciency PART OF TheOREM 2.3. Our assumptions are that $\Gamma$ is uniformly discrete and that $D^{-}\left(\Gamma_{v}\right)=\beta / \pi>\alpha / \pi$. The assertion is that then $\Gamma_{v}$ is a set of sampling. Suppose this is not true. Then by Lemma 4.3 there is a $\Gamma_{v_{0}}^{(0)} \in W\left(\Gamma_{v}\right)$ such that $S\left(\Gamma_{v_{0}}^{(0)}\right) f=0$ for some nonzero function $f \in F_{\alpha}^{\infty}$. We may assume $0 \notin \Gamma^{(0)}, f(0) \neq 0$, and $\|f\|_{\infty}=1$. Jensen's formula yields

$$
\begin{aligned}
\log |f(0)| & \leqq \sum_{z_{j} \in \Gamma^{(0)} \cap D(0, r)} v_{0}\left(z_{j}\right) \log \frac{\left|z_{j}\right|}{r}+\frac{1}{2 \pi} \int_{0}^{2 \pi} \log \left|f\left(r e^{i \theta}\right)\right| d \theta \\
& \leqq-\int_{0}^{r} \frac{n(\rho)}{\rho} d \rho+\frac{\alpha}{2} r^{2} .
\end{aligned}
$$

where $n(\rho)$ denotes the number of points from $\Gamma_{v_{0}}^{(0)}$ in the disk $|z|<\rho$. Let $\varepsilon=(\beta-\alpha) / 2$. According to the definition of lower uniform density there is a positive number $r_{\varepsilon}$ such that $n(\rho)>\rho^{2}(\beta-\varepsilon)$ whenever $\rho>r_{\varepsilon}$. Thus from (12) we find that

$$
\log |f(0)| \leqq-\frac{\varepsilon}{2} r^{2}+\frac{\beta-\varepsilon}{2} r_{\varepsilon}^{2} .
$$

Letting $r \rightarrow \infty$ we obtain a contradiction. We conclude that $\Gamma_{v}$ is a set of sampling.

\section{Proof of Theorem 2.4.}

The following lemma follows from Lemma 5.1 in [11] since clearly $\Gamma$ is a set of interpolation if $\Gamma_{v}$ is a set of interpolation.

LEMMA 5.1. Every set of interpolation for $F_{\alpha}^{\infty}$ is uniformly discrete.

Lemma 5.2. $\Gamma_{v_{j}}^{(j)} \rightarrow \Gamma_{v}$ implies $N_{\infty}\left(\Gamma_{v}\right) \leqq \lim \inf N_{\infty}\left(\Gamma_{v_{j}}^{(j)}\right)^{\text {. }}$

Proof. We may assume that the right-hand side is bounded, and even that $\sup \left|N_{\infty}\left(\Gamma_{v_{j}}^{(j)}\right)\right|<\infty$ by picking an appropriate subsequence. The result then follows by the compactness property. 
Proof of the necessity. We define

$$
\rho_{\infty}\left(z, \Gamma_{v}\right)=\sup e^{-\frac{\alpha}{2}|z|^{2}}|f(z)|,
$$

where the supremum is taken over all functions $f$ for which $S\left(\Gamma_{v}\right) f=0$ and $\|f\|_{\infty} \leqq 1$. We may now prove an analogue of Lemma 5.6 in [11] for $\rho_{\infty}(\cdot, \cdot)$ and finish the proof of the necessity part of the theorem along the same lines as in Section 5 in [11]; we omit the straightforward details.

Proof of the sufficiency. As in [14], we may assume that $\Gamma_{v}$ is uniformly close to a square lattice $\Lambda$ of density $\beta / \pi$, with $\beta<\alpha$ and $D^{+}\left(\Gamma_{v}\right)=\beta / \pi$. Let $g_{j}$ denote the function $g$ in (9) associated with $\Gamma_{v}-z_{j}$, and put $n=v\left(z_{j}\right)$. In order to construct an interpolation formula, we define first

$$
\psi_{j, k}(z)=\left(\frac{\alpha}{k !}\right)^{\frac{1}{2}} \frac{g_{j}(z)}{z^{n+1-k}} .
$$

We observe that

$$
\left\langle\psi_{j, k}, T_{z_{i}} f_{m}\right\rangle= \begin{cases}1, & j=i, k=m \\ 0, & j=i, m<k \text { or } j \neq i, m<v\left(z_{i}\right) .\end{cases}
$$

If we define

$$
\begin{aligned}
& \phi_{j, n}=\psi_{j, n} \\
& \phi_{j, k}=\psi_{j, k}-\sum_{m=k+1}^{n}\left\langle\psi_{j, k}, T_{z_{j}} f_{m}\right\rangle \phi_{j, k}, \quad k=n-1, \ldots, 0,
\end{aligned}
$$

we have therefore

$$
\left\langle\phi_{j, k}, f_{m}^{z_{i}}\right\rangle= \begin{cases}1, & j=i, k=m \\ 0, & \text { otherwise. }\end{cases}
$$

From Lemma 3.3 it is easy to see that these functions enjoy estimates of the form

$$
e^{-\frac{\beta}{2}|z|^{2}}\left|\phi_{j, k}(z)\right| \leqq C e^{c\left|z-z_{j}\right| \log \left|z-z_{j}\right|},
$$

with $c$ as in Lemma 3.3 and $C$ a constant depending on $Q, q, \beta$, and $n$. For given $a=\left\{a_{j}^{(k)}\right\} \in l^{\infty}$, we may interpolate with help of the formula

$$
f(z)=\sum_{j} \sum_{k=0}^{v\left(z_{j}\right)-1} a_{j}^{(k)} \phi_{j, k}(z),
$$

which by some straightforward estimates (using (14)) provides a solution to the interpolation problem; see [14] for details. 


\section{Proofs in the $L^{2}$ case.}

We outline the proofs of the $L^{2}$ theorems.

LEMMA 6.1. There exists a positive constant B such that

$$
\sum_{z \in \Gamma} \sum_{k=0}^{v(z)-1}\left|\left\langle f, T_{z} f_{k}\right\rangle\right|^{2} \leqq B\|f\|_{2}^{2}
$$

for all $f \in F_{\alpha}^{2}$ if and only if $\Gamma$ can be expressed as a finite union of uniformly discrete sets.

Proof. The sufficiency follows from (7) and the necessity is a consequence of Lemma 7.1 in [11].

LEMMA 6.2. If $\Gamma$ is a set of sampling for $F_{\alpha}^{2}$, then $\Gamma$ contains a uniformly discrete set that is also a set of sampling.

Proof. For $\varepsilon>0$ we construct (as we may) a uniformly discrete subset $\Gamma^{\prime}$ of $\Gamma$ such that $d\left(\zeta, \Gamma^{\prime}\right)<\varepsilon$ for each $\zeta \in \Gamma$. We have then $\Gamma=\cup_{\zeta^{\prime} \in \Gamma^{\prime}}\left(\Gamma \cap D\left(\zeta^{\prime}, \varepsilon\right)\right)$. By the preceding lemma there is a uniform bound, say $P$, on the number of points in $\Gamma \cap D\left(\zeta^{\prime}, \varepsilon\right), \varepsilon \leqq 1 / 2$.

Proceeding as in the proof of Lemma 3.1 we easily deduce the estimate

$$
\left|K f, T_{\zeta} f_{k}\right\rangle|-|\left\langle f, T_{\zeta^{\prime}} f_{k}\right\rangle|| \leqq C\left|\zeta-\zeta^{\prime}\right| \iint_{D(\zeta, 1)}|f(z)|^{2} d \mu_{\alpha}(z),
$$

which holds for, say, $\left|\zeta-\zeta^{\prime}\right| \leqq 1 / 2, C$ depending only on $\alpha$ and $k$. We square this inequality and sum over $\Gamma_{v}$ (for each $\zeta \in \Gamma$ we pick some point $\zeta^{\prime} \in \Gamma^{\prime} \cap D(\zeta, \varepsilon)$ ):

$$
\begin{aligned}
\sum_{\zeta \in \Gamma} \sum_{k=0}^{v(\zeta)-1}||\left\langle f, T_{\zeta} f_{k}\right\rangle|-|\left\langle f, T_{\zeta^{\prime}} f_{k}\right\rangle||^{2} & \leqq C \varepsilon^{2} \sum_{\zeta \in \Gamma} \sum_{v(\zeta)-1} \iint_{D(\zeta, 1)}|f(z)|^{2} d \mu_{\alpha}(z) \\
& \leqq \max (v) P C \varepsilon^{2}\|f\|_{2}^{2} .
\end{aligned}
$$

This gives us

$$
\left(\sum_{\zeta \in \Gamma} \sum_{k=0}^{v(z)-1}\left|\left\langle f, T_{\zeta} f_{k}\right\rangle\right|^{2}\right)^{\frac{1}{2}}-\left(\sum_{\zeta \in \Gamma} \sum_{k=0}^{v(z)-1}\left|\left\langle f, T_{\zeta^{\prime}} f_{k}\right\rangle\right|^{2}\right)^{\frac{1}{2}} \leqq \varepsilon \sqrt{\max (v) P C}\|f\|_{2},
$$

and hence

$$
\left\|S\left(\Gamma_{v}\right) f\right\|_{2} \leqq P\left\|S\left(\Gamma_{v}^{\prime}\right) f\right\|_{2}+C \varepsilon\|f\|_{2} .
$$

The proof is finished since the choice of $\varepsilon$ is at our disposal.

We omit the proof of the next lemma; see Lemma 7.3 in [11] and Lemma 5.2 in this paper. 
LEMma 6.3. If $\Gamma_{v_{j}}^{(j)} \rightarrow \Gamma_{v}, \inf _{j} q\left(\Gamma^{(j)}\right)>0$, and $\sup _{j} \max \left(v_{j}\right)<\infty$, then $\mathrm{M}_{2}\left(\Gamma_{v}\right) \leqq$ $\liminf M_{2}\left(\Gamma_{v_{j}}^{(j)}\right)$.

LEMMA 6.4. Suppose $\Gamma_{v}$ is a set of sampling for $F_{\alpha}^{2}$. If any point from $\Gamma_{v}$ is removed (thus reducing the multiplicity of one of the points of $\Gamma$ by one), the sequence remains a set of sampling for $F_{\alpha}^{2}$.

Proof. In view of Lemma 6.2, we may assume that $\Gamma$ is uniformly discrete. It is easy to see that we must have $D^{-}\left(\Gamma_{v}\right)>0$; in fact, by some arguments from [11] we know that $D^{-}\left(\Gamma_{v}\right) \geqq \alpha / \pi$. This implies that $D^{-}(\Gamma)>0$ by the assumption that $\max (v)<\infty$. Since the removal of a vector from a frame leaves either a frame or an incomplete set (see [5, p. 360]), we need to show that $\Gamma_{v}$ remains a set of uniqueness after one point has been removed. Assume this is not true. Then for some $\zeta_{0} \in \Gamma$ there is a function $g_{0} \in F_{\alpha}^{2}$ with

$$
g_{0}^{(k)}(z)= \begin{cases}1, & z=\zeta_{0}, k=v\left(\zeta_{0}\right)-1 \\ 0, & z \in \Gamma \backslash\left\{\zeta_{0}\right\}, k<v(z)\end{cases}
$$

We put $g(z)=\left(z-\zeta_{0}\right) g_{0}(z)$, and observe that clearly $g(z) /(z-\zeta) \in F_{\alpha}^{2}$ for arbitrary $\zeta \in \Gamma$. We then argue as in the proof of Lemma 6.2 in [13] in order to obtain a contradiction.

The following lemma is an immediate consequence.

LEMMA 6.5. If $\Gamma_{v}$ is a set of interpolation for $F_{\alpha}^{2}$, there is a nonzero function in $g \in F_{\alpha}^{2}$ with $S\left(\Gamma_{v}\right) g=0$.

Proof. A set of interpolation that is also a set of uniqueness, is necessarily a set of sampling. This is impossible in view of the preceding lemma.

Proof of the necessity part of Theorem 2.1. We argue as in [13]: Consider, by Lemma 6.2, a uniformly discrete set $\Gamma$ for which $\Gamma_{v}$ is a set of sampling for $F_{\alpha}^{2}$. Lemma 6.3 and the fact that all sets in $W\left(\Gamma_{v}\right)$ are uniformly discrete imply that $W\left(\Gamma_{v}\right)$ consists only of sets of sampling. By Lemma 6.4 we have that every set of sampling for $F_{\alpha}^{2}$ is a set of uniqueness for $F_{\alpha}^{\infty}$. For suppose $\Gamma_{v}$ is a set of sampling for $F_{\alpha}^{2}$ and that $S\left(\Gamma_{v}\right) g=0$ for some $g \in F_{\alpha}^{\infty}$. Then the function

$$
f(z)=\frac{g(z)}{\left(z-z_{1}\right)\left(z-z_{2}\right)},
$$

$z_{1}, z_{2} \in \Gamma$, belongs to $F_{\alpha}^{2}$ and has zeros of order at least $v(z)$ at every point $z$ of $\Gamma$ except at $z_{1}$ and $z_{2}$, where the zeros are of order at least $v\left(z_{1}\right)-1$ and $v\left(z_{2}\right)-1$, respectively. This contradicts Lemma 6.4.

Thus every set in $W\left(\Gamma_{v}\right)$ is a set of uniqueness for $F_{\alpha}^{\infty}$. It follows from Lemma 
4.3 that $\Gamma_{v}$ is also a set of sampling for $F_{\alpha}^{\infty}$, and thus by Theorem 2.3, $D^{-}\left(\Gamma_{v}\right)>\alpha / \pi$.

Proof of the necessity part of Theorem 2.2. For $z \in \mathrm{C}$ we now define

$$
\rho_{2}\left(z, \Gamma_{v}\right)=\sup _{f} e^{-\frac{\alpha}{2}|z|^{2}}|f(z)|
$$

where $f$ ranges over those functions $f$ for which $S\left(\Gamma_{v}\right) f=0$ and $\|f\|_{2} \leqq 1$. With help of this notion and Lemma 6.5 we may proceed as in Section 7 of [13]. We omit the straightforward details.

Proof of the sufficiency part of Theorem 2.1. In view of Lemma 6.1, we need to show that there exists a positive constant $A$ so that

$$
\left\|S\left(\Gamma_{v}\right)\right\|_{2}^{2} \geqq A\|f\|_{2}
$$

for all $f \in F_{\alpha}^{2}$. We could have applied the techniques of [14], but we will give an alternate argument which is less computational and of a somewhat more general nature. Similar considerations appear in Section 6 in [12].

Let $F_{\alpha}^{\infty, 0}=\left\{g \in F_{\alpha}^{\infty}: e^{-\frac{\alpha}{2}|z|^{2}}|g(z)| \rightarrow 0\right.$ as $\left.|z| \rightarrow 0\right\} ; F_{\alpha}^{\infty, 0}$ is clearly a closed subspace of $F_{\alpha}^{\infty}$. Suppose $\Gamma$ is uniformly discrete and $D^{-}\left(\Gamma_{v}\right)=\beta / \pi>\alpha / \pi$. Then $\Gamma_{v}$ is a set of sampling for $F_{\alpha+\varepsilon}^{\infty}$ if, say, $\varepsilon=(\beta-\alpha) / 2$. This means that the transformation $S_{\alpha+\varepsilon}\left(\Gamma_{v}\right) f$ is a bounded invertible mapping from $F_{\alpha+\varepsilon}^{\infty, 0}$ onto a closed subspace of the sequence space $c_{0}$; denote this subspace by $a_{0}$. Then any bounded linear functional $\phi$ on $F_{\alpha+\varepsilon}^{\infty, 0}$ induces a bounded linear functional on $a_{0}$ by

$$
\tilde{\phi}(\xi)=\phi\left(S_{\alpha+\varepsilon}\left(\Gamma_{v}\right)^{-1} \xi\right),
$$

with $\|\tilde{\phi}\| \leqq M_{\infty}\left(\Gamma_{v}\right)\|\phi\|$. For arbitrary $\zeta \in \mathrm{C}$ we let $\phi_{\zeta}$ denote the normalized functional of point evaluation at $\zeta$, i.e.,

$$
\phi_{\zeta}(f)=e^{-\frac{\alpha+\varepsilon}{2}|\zeta|^{2}} f(\zeta)
$$

Trivially, $\left\|\phi_{\zeta}\right\|=1$. By the above reasoning, and since the dual space of $c_{0}$ is $l^{1}$, there exists for each $\zeta$ a sequence of numbers $\left\{g_{j, k}(\zeta)\right\}$ such that

$$
e^{-\frac{\alpha+\varepsilon}{2}|\zeta|^{2}} f(\zeta)=\sum_{z_{j} \in \Gamma} \sum_{k=0}^{v\left(z_{j}\right)-1} g_{j, k}(\zeta)\left\langle f, T_{z_{j}}^{\alpha+\varepsilon} f_{k}\right\rangle_{\alpha+\varepsilon}
$$

with

$$
\sum_{z j \in \Gamma} \sum_{k=0}^{v\left(z_{j}\right)-1}\left|g_{j, k}(\zeta)\right| \leqq M=M_{\infty}\left(\Gamma_{v}, \alpha+\varepsilon\right)
$$

Now let $f \in F_{\alpha}^{2}$. We apply (17) to the function

$$
f_{\zeta, \varepsilon}(z)=e^{\varepsilon\left(\overline{\zeta z}-|\zeta|^{2}\right)} f(z)
$$


which is seen to belong to $F_{\alpha+\varepsilon}^{\infty, 0}$. Thus,

$$
e^{-\frac{\alpha+\varepsilon}{2}|\zeta|^{2}} f(\zeta)=\sum_{z_{j} \in \Gamma} \sum_{k=0}^{v\left(z_{j}\right)-1} g_{j, k}(\zeta)\left\langle f_{\zeta, \varepsilon}, T_{z_{j}}^{\alpha+\varepsilon} f_{k}\right\rangle_{\alpha+\varepsilon}
$$

A little computation shows that

$$
\left\langle f_{\zeta, \varepsilon}, T_{z_{j}}^{\alpha+\varepsilon} f_{k}\right\rangle_{\alpha+\varepsilon}=e^{-\frac{\varepsilon}{2}\left(|\zeta|^{2}+\left|\zeta-z_{j}\right|^{2}-\left(\overline{\zeta z_{j}}-\overline{\left.\zeta z_{j}\right)}\right)\right.}\left\langle h, f_{k}\right\rangle_{\alpha+\varepsilon}
$$

where

$$
h(z)=e^{\varepsilon\left(\overline{\left.\zeta-z_{j}\right) z}\right.}\left(T_{-z_{j}}^{\alpha} f\right)(z) .
$$

Using (3) twice we find that

$$
\left\langle h, f_{k}\right\rangle_{\alpha+\varepsilon}=\left((\alpha+\varepsilon)^{k} k !\right)^{-\frac{1}{2}} \sum_{i=0}^{k}\left(\begin{array}{l}
k \\
l
\end{array}\right) \varepsilon^{k-l}\left(\overline{\zeta-z_{j}}\right)^{k-j}\left(\alpha^{l} l !\right)^{\frac{1}{2}}\left\langle f, T_{z_{j}}^{\alpha} f_{l}\right\rangle_{\alpha} .
$$

Hence

$$
\left|\left\langle f_{\zeta, \varepsilon}, T_{z_{j}}^{\alpha+\varepsilon} f_{k}\right\rangle_{\alpha+\varepsilon}\right| \leqq C(k, \alpha, \varepsilon) e^{-\frac{\varepsilon}{2}\left(|\zeta|^{2}+\left|\zeta-z_{j}\right|^{2}\right)} \sum_{i=0}^{k}\left|\zeta-z_{j}\right|^{k-l}\left|\left\langle f, T_{z_{j}}^{\alpha} f_{l}\right\rangle_{\alpha}\right| .
$$

Inserting this into (18) and applying the Cauchy-Schwarz inequality to the sum, we obtain

$$
e^{-\alpha|\zeta|^{2}}|f(\zeta)|^{2} \leqq C M^{2} \sum_{z_{j} \in \Gamma} \sum_{k=0}^{v\left(z_{j}\right) \rightarrow 1} e^{-\varepsilon\left|\zeta-z_{j}\right|^{2}} \sum_{l=0}^{k}\left|\zeta-z_{j}\right|^{2(k-l)}\left|\left\langle f, T_{z_{j}} \alpha f_{l}\right\rangle_{\alpha}\right|^{2} .
$$

We integrate with respect to area measure over $C$ and arrive at the required estimate.

Proof of the sufficiency part of Theorem 2.2. We apply formula (15) and proceed as in the corresponding part in [14].

\section{REFERENCES}

1. G. Battle, Heisenberg proof of the Balian-Low theorem, Lett. Math. Phys. 15 (1988), 175-177.

2. S. Bergman, The Kernet Function and Conformal Mapping, Math. Surveys V, New York, 1950.

3. A. Berling, The Collected Works of Arne Beurling, Vol 2 Harmonic Analysis (ed. L. Carleson, P. Malliavin, J. Neuberger and J. Werner), Birkhäuser, Boston, 1989, 341-365.

4. I. Daubechies, Time-frequency localization operators - a geometric phase space approach, IEEE Trans. Inform. Theory 34 (1988), 605-612

5. R. J. Duffin and A. C. Schaeffer, A class of nonharmonic Fourier series, Trans. Amer. Math. Soc. 72 (1952), 341-366.

6. G. B. Folland, Harmonic Analysis in Phase Space, Princeton Univ. Press, Princeton, 1989.

7. K. Gröchenig and D. Walnut, $A$ Riesz basis for the Bargmann-Fock space related to sampling and interpolation, Ark. Mat. 30 (1992), 283-295.

8. K. Hoffman, Banach Spaces of Analytic Functions, Prentice-Hall, Englewood Cliffs, 1962. 
9. A. J. Jerri, The Shannon sampling theorem - its various extensions and applications: A tutorial review, Proc. IEEE 65 (1977), 1565-1596.

10. H. J. Landau. Necessary density conditions for sampling and interpolation of certain entire functions, Acta Math. 117 (1967), 37-52.

11. K. Seip, Reproducing formulas and double orthogonality in Bargmann and Bergman spaces, SIAM J. Math. Anal. 22 (1991), 856-876.

12. K. Seip, Beurling type density theorems in the unit disk, Invent. Math., to appear.

13. K. Seip, Density theorems for sampling and interpolation in the Bargmann-Fock space I, J. Reine Angew. Math. 429 (1992), 91-106.

14. K. Seip and R. Wallstén, Density theorems for sampling and interpolation in the Bargmann-Fock space II, J. Reine Angew. Math. 429 (1992), 107-113.

15. A. Unterberger, Les opérateurs métadifférentiels, in Lecture Notes in Phys. 126 (1980), 205-241.

16. K. Øyma, Interpolation in $H^{p}$-spaces, Proc. Amer. Math. Soc. 76 (1979), 81-88. 